doi: $10.2306 /$ scienceasia1513-1874.2012.38.356

\title{
Ceftriaxone improves spatial learning and memory in chronic cerebral hypoperfused rats
}

\author{
Phanit Koomhin, Kanokwan Tilokskulchai, Sompol Tapechum* \\ Department of Physiology, Faculty of Medicine Siriraj Hospital, Mahidol University, Bangkok \\ *Corresponding author, e-mail: sompol.tap@mahidol.ac.th
}

Received 11 May 2012

Accepted 24 Sep 2012

\begin{abstract}
Cerebral hypoperfusion is associated with cognitive decline in ageing, mild cognitive impairment, vascular type dementia, and Alzheimer's disease. The mechanisms leading to such neurological impairments are still uncertain. Although several mechanisms have been proposed as contributing factors leading to neuronal injury, glutamate excitotoxicity seems to be the relevant one. Recently, it was found that $\beta$-lactam antibiotics such as ceftriaxone show a neuroprotective effect by upregulation of glutamate transporter and reduction of glutamate excitotoxicity. To study the contribution of glutamate excitotoxicity and the effects of ceftriaxone on spatial learning and memory in chronic cerebral hypoperfusion, we conducted experiments in rats subjected to permanent bilateral common carotid artery occlusion. Ceftriaxone (200 mg/kg) was injected daily in rats for 5 days after the onset of the arterial ligation. A Morris water maze was used to assess learning and memory two months after arterial ligation. Hippocampal histology and glutamate transporter 1 (GLT-1) expression were also studied. Results showed that ceftriaxone improved learning and memory performance. Consistently, the histological study showed an increase hippocampal CA1 and CA3 neuronal numbers in the ceftriaxone-treated group compared with the vehicle-treated group. Although not statistically significant, the GLT-1 protein level in the hippocampus was $86 \%$ higher than the sham group. We conclude that ceftriaxone treatment can attenuate neuronal injury and improve spatial learning and memory after chronic cerebral hypoperfusion and that glutamate excitotoxicity may play an important role in the pathophysiology of chronic cerebral hypoperfusion.
\end{abstract}

KEYWORDS: glutamate transporter, glutamate excitotoxicity, hippocampus

\section{INTRODUCTION}

Reduction of cerebral blood flow (CBF) results in neuronal injury and neurological impairment which depends on degree and duration of the insult ${ }^{1}$. The condition can occur focally or globally ${ }^{2}$. Complete cessation of blood flow as occur in stroke causes cerebral infarction while cerebral hypoperfusion usually results in selective injury of neurons in vulnerable areas of the brain. Cerebral hypoperfusion is observed in ageing, mild cognitive impairment, vascular type dementia and Alzheimer's disease ${ }^{3-5}$. In animal, cerebral hypoperfusion can be induced by occluding major blood vessels supplying the brain. Bilateral common carotid artery occlusion (2VO) is among several vascular occlusion models being used extensively in the study of chronic cerebral hypoperfusion. Permanent ligation of the vessels causes incomplete reduction of blood flow to $35-45 \%$ of control level in cortex and white matter regions and approximately $60 \%$ of control level in hippocampus. The hypoperfused state is maintained for duration up to 3 months after the ligation $^{6}$. Chronic reduction of $\mathrm{CBF}$ in this manner has been previously thought to be innocuous to neurons ${ }^{7}$. However, $2 \mathrm{VO}$ alters brain energy metabolism, induces progressive neuronal cell death, and finally leads to cognitive impairment ${ }^{8-10}$. Neuronal injury is not detected initially but it occurred in a delayed manner. There are several mechanisms leading to neuronal injury and death after insufficient CBF. Previous studies showed that oxidative stress plays a key role in chronic cerebral hypoperfusion ${ }^{11,12}$. Nevertheless, other mechanisms including glutamatemediated excitotoxicity, acidotoxicity, and inflammation are thought to participate in the process of neuronal injury ${ }^{13}$. The role of glutamate excitotoxicity has been demonstrated in focal ischaemic model ${ }^{14}$ and several neurodegenerative diseases ${ }^{15-17}$. However, further detailed study about glutamate excitotoxicity involvement in chronic cerebral hypoperfusion has yet to be investigated.

Glutamate signalling is essential for learning and memory processes ${ }^{18}$. Glutamate is released from glutamatergic synapses in regulated fashion ${ }^{19}$. More than $90 \%$ of extracellular glutamate is removed by glial glutamate transporter (GLT-1), and glutamate- 
aspartate transporter (GLAST), specifically expressed in astrocytes ${ }^{20,21}$. In ischaemic condition, excessive glutamate release occurs. It results in overstimulation of glutamate receptors which consequently raises cytosolic $\mathrm{Ca}^{2+}$ concentration to the detrimental level. The $\mathrm{Ca}^{2+}$ overload finally triggers cell death ${ }^{22-24}$. This process is so-called glutamate excitotoxicity. In 2005, Rothstein found that ceftriaxone (CTX) upregulated glutamate transporters and showed neuroprotective effect ${ }^{25}$. Ceftriaxone is a $\beta$-lactam antibiotic which is one of the members of third generation cephalosporins. It is readily transported across bloodbrain barrier and is effective against gram negative and gram positive bacteria through the inhibition of cell wall synthesis ${ }^{26}$. Pre and post treatment of CTX reduced infarct volume after middle cerebral artery occlusion, an in vivo model of stroke ${ }^{27,28}$. It enhances both protein expression and functional activity of GLT- 1 by mechanism involving NF- $\mathrm{KB}$ signalling pathway ${ }^{29}$. These observations suggest the high efficacy of CTX in the upregulation of GLT-1 and the reduction of glutamate excitotoxicity. For $2 \mathrm{VO}$ model, glutamate excitotoxicity may also cause neuronal injury after the chronic $\mathrm{CBF}$ reduction. Whether injury via glutamate excitotoxicity taking part in behavioural impairment at 8 weeks of chronic hypoperfusion needs further investigation. To emphasize the importance of glutamate-mediated toxicity in this condition, we carried out experiments examining the effects of ceftriaxone treatment in chronic cerebral hypoperfused rats. Spatial learning and memory, hippocampal histology and glutamate transporter expression were studied in chronic cerebral hypoperfused rats with and without ceftriaxone treatment.

\section{MATERIALS AND METHODS}

\section{Animals}

A total of 84 young adult male Spraque Dawley rats, 8 weeks of age, were used in the experiments. They were divided into 3 groups according to vessel ligation and treatments: the sham-operated group (sham), the vehicle-treated bilateral common carotid artery-occluded group (2VO-Veh), and the ceftriaxone-treated bilateral common carotid arteryoccluded group (2VO-CTX).

All animals were obtained from the National Laboratory Animal Centre, Mahidol University, Salaya, Nakhon Pathom. They were maintained in ventilated isolation cages under natural light/dark cycle and constant room temperature of $25^{\circ} \mathrm{C}$. The rats were allowed to freely access to standard diet and water throughout the experimental period. Experimental procedures were approved by Siriraj Animal Care and Use Committee, Faculty of Medicine Siriraj Hospital, Mahidol University, Thailand (protocol number: SI-ACUP 001/2554, approval date: 27 January 2011).

\section{Surgical procedure}

The operation was conducted as previously described with slight modification ${ }^{30,31}$. The rats were anaesthetized with ketamine $60 \mathrm{mg} / \mathrm{kg}$ and xylazine $6 \mathrm{mg} / \mathrm{kg}$ intramuscularly. After induction of anaesthesia, a ventral cervical incision of neck was performed. The right and left common carotid arteries were gently separated from carotid sheath and vagus nerves. The arteries were then permanently ligated with silk suture. The sham-operated animals went through the same procedure except that the arteries were not ligated. Animals were allowed to fully recover before returning to their cages. All surgical procedures were carried out under sterile techniques. The rat body temperature was maintained using a heating lamp during surgery and recovery.

\section{Drug administration}

Ceftriaxone (purchased from Siam Bheasach, Thailand) was diluted in sterile water to the concentration of $25 \%(\mathrm{w} / \mathrm{v})$. Ceftriaxone $(200 \mathrm{mg} / \mathrm{kg})$ or vehicle were intraperitoneally injected once daily for 5 days starting on the first day after surgery.

\section{Morris water maze test}

Morris water maze test was conducted 8 weeks after surgery in sham $(n=12)$, 2VO-Veh $(n=14)$, and 2VO-CTX $(n=13)$. The protocol was slightly modified from the previous studies ${ }^{32,33}$. The Morris water maze is a circular swimming pool (diameter $200 \mathrm{~cm}$, height $50 \mathrm{~cm}$ ) located in a room with various extra-maze cues. The pool was filled with water to the depth of $25 \mathrm{~cm}$. The glass escape platform was submerged $2 \mathrm{~cm}$ below water surface and located at the centre of one quadrant. A video camera was fixed to the ceiling of the room and was connected to a computer for recording.

In the hidden platform session, the rats were allowed to swim for $120 \mathrm{~s}$ to find the hidden platform. The latencies to find the hidden platform of each rat were measured 4 trials per day for 5 days. The location of the hidden platform was placed in the same quadrant every single day. The probe trial was performed on the 6th day. During the probe trial, the platform was removed and the rats were allowed to swim for $120 \mathrm{~s}$. The time spent in the target quadrant was recorded. 
The visible platform session was also conducted at 8 weeks after surgery to evaluate the visual performance, sensorimotor performance and motivation to perform the task. Separated groups of animals (sham: $n=8$, 2VO-Veh: $n=9$, and 2VO-CTX: $n=10$ ) were used to prevent the carry on effects on Morris water maze test. The platform was raised $2 \mathrm{~cm}$ higher than the water surface and was tied with two 40-mm balls as visible cues. The swimming session was similar to the hidden platform but the location of the platform was moved to different quadrants in different trials. Average swimming speeds were calculated from the first trial of the test. The average swimming speeds and the mean escape latencies were analysed.

\section{Histological assessment}

Twenty-four hours after the last session of the behavioural test, the rats were deeply anaesthetized with ketamine/xylazine. The rats were then transcardially perfused with $200 \mathrm{ml}$ saline solution and then followed by $4 \%$ paraformaldehyde in $0.1 \mathrm{M}$ phosphate buffer, $\mathrm{pH}$ 7.4. The brains were removed out and were post-fixed in $4 \%$ paraformaldehyde solution at $4{ }^{\circ} \mathrm{C}$ for $24 \mathrm{~h}$. After post-fixation, the brains were processed in tissue processor and were embedded in paraffin. Coronal sections of $10 \mu \mathrm{m}$ thickness were prepared using a microtome. The sections were mounted on 3\% APES-coated slides. To investigate neuronal damage, the slides were stained with cresyl violet and mounted with Permount. The stained slides were examined under a light microscope (Carl Zeiss Axio Imager M2, Germany). Pictures of hippocampus were taken at magnification of $200 \times$ for neuronal counting. For each brain, series of 3 sections spaced at an interval of $200 \mu \mathrm{m}$ from each other were analysed. For each slide, the total numbers of live neurons in an area of $530 \mu \mathrm{m} \times 700 \mu \mathrm{m}$ (approximately $0.37 \mathrm{~mm}^{2}$ ) of hippocampal CA1 and CA3, $250 \mu \mathrm{m} \times 100 \mu \mathrm{m}$ (approximately $0.25 \mathrm{~mm}^{2}$ ) of dentate gyrus were counted by an investigator who was blinded to the experimental groups. The live neurons were identified by their large nuclei with prominent nucleoli.

\section{Western blot analysis}

Eighteen rats were divided into 3 groups equally and sacrificed by decapitation at the first week after ischaemic insult. Right hippocampal region was quickly dissected out and homogenized in RIPA buffer containing protease inhibitor cocktails. The homogenate was centrifuged at $14000 \mathrm{~g}$ at $4{ }^{\circ} \mathrm{C}$ for $10 \mathrm{~min}$. The supernatant was used for protein analysis. The protein concentration of the supernatant was determined using the Bradford assay. For gel elec- trophoresis, each lane was loaded with approximately $30 \mu \mathrm{g}$ of protein. After separated by 4-10\% SDS polyacrylamide gel electrophoresis, protein on the gel was then electrophoretically transferred to nitrocellulose membranes. The membranes were blocked and incubated overnight with antibody generated against the N-terminus of GLT-1. After washing, the membrane was incubated with an alkaline phosphataseconjugated secondary antibody for $1 \mathrm{~h}$ and then was incubated with BCIP/NBT. The band intensities were analysed and were normalized with $\beta$-actin levels.

\section{Statistical analysis}

Results were expressed as mean \pm SEM. The differences of mean escape latencies from Morris water maze test were analysed using repeated-measure ANOVA with groups (sham, 2VO-Veh, 2VO-CTX) as grouping factor and trial days as repeated factors. Bonferroni post hoc test was used for pairwise comparison. The other data were analysed using oneway ANOVA. Fisher's least significant difference post hoc test was used to determine the difference between groups. Statistical significances were accepted at $p<$ 0.05 .

\section{RESULTS \\ Effects of ceftriaxone on learning and memory performance}

The mean escape latencies were different among groups as reported by repeated-measure ANOVA $(F(2,36)=18.017, p<0.001)$. Bilateral occlusion of common carotid arteries caused learning and memory deficit (Fig. 1a). The mean escape latencies in the $2 \mathrm{VO}-\mathrm{Veh}$ were significantly longer than those of Sham $(p<0.01)$. Treatment with ceftriaxone (2VO-CTX) improved spatial performance as mean escape latencies were shorter compared with $2 \mathrm{VO}$ Veh $(p<0.05)$. The swimming speeds were not different between groups (Fig. 1b), indicating that the motor performance was not affected in this model. Memory retention was tested in probe trial. The rats were allowed to swim for $120 \mathrm{~s}$ to test their spatial bias or memory of the platform position in the target quadrant. There was difference among groups $(F(2,36)=9.730, p<0.001) .2$ VO-Veh group spent less time in the target quadrant compared with Sham group. 2VO-CTX group spent more time in the target quadrant than $2 \mathrm{VO}-\mathrm{Veh}$ group $(p<0.05)$ which referred to better memory retention in the ceftriaxonetreated rats.

To evaluate visual performance, sensorimotor performance and motivation to perform the task, the 

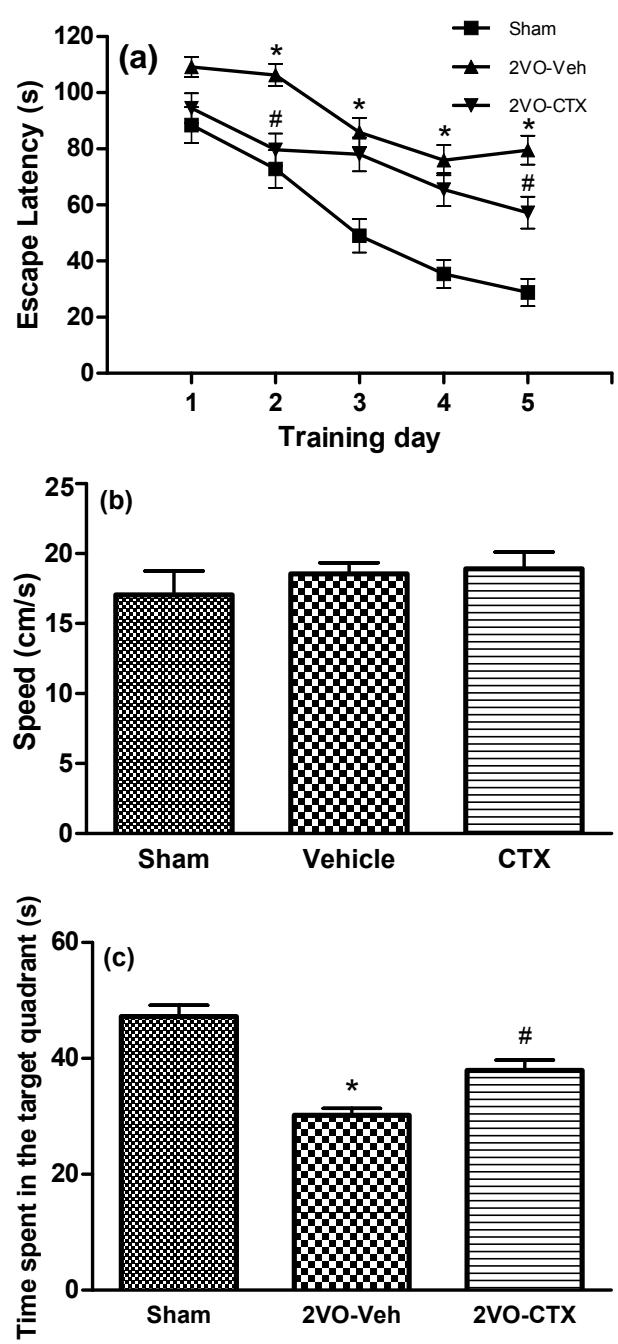

Fig. 1 Effects of ceftriaxone on spatial learning and memory performance in chronic cerebral hypoperfused rats using Morris water maze. (a) Mean escape latencies from training day 1-5 represent daily spatial learning performance. (b) Swimming speed from the first trial of the Morris water maze test represents motor functions. (c) Time spent in the target quadrant during probe test without the submerged platform represents memory performance. Sham: $n=12$, 2VO-Veh: $n=14$, and 2VO-CTX: $n=13$ were used in the hidden platform session. Data present as mean \pm SEM. ${ }^{*} p<0.05$ versus sham-operated group. ${ }^{\#} p<0.05$ versus 2VO-Veh group.

visible platform session of Morris water maze was tested in separated groups of rats (Fig. 2). The rats were subjected to find the visible platform that was randomly changed for each trial. There was no difference among groups in visible-platform session $(F(2,24)=1.630, p=0.217)$. The average
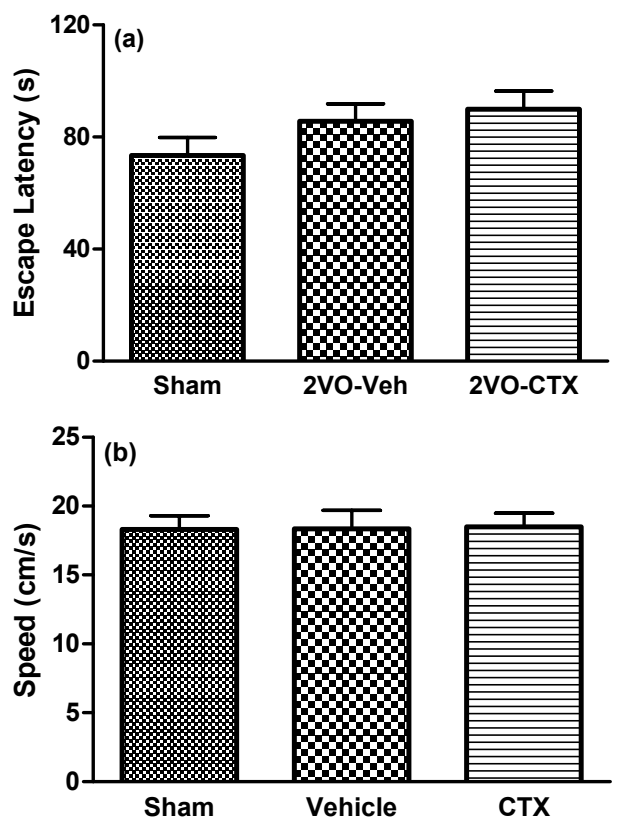

Fig. 2 (a) Mean escape latencies obtained from visible session of Morris water maze task. (b) Average swimming speeds calculated from the first trial of the visible session. Sham: $n=8$, 2VO-Veh: $n=9$, 2VO-CTX: $n=10$ were used in this task. Data present as mean \pm SEM.

swimming speeds were also not different among rats $(F(2,24)=0.007, p=0.993)$.

\section{Effects of ceftriaxone on hippocampal neurons}

After 8 weeks of bilateral common carotid artery occlusion, the number of survival neurons in hippocampal CA1 $(F(2,42)=7.154, p=0.009)$ and hippocampal CA3 $(F(2,42)=3.282, p=0.047)$ were significantly reduced (Fig. 3a, 2VO-Veh versus Sham, $p=0.003$ ). More dead neurons, seen as pyknotic cells, were also observed in the cresyl-stained sections of hippocampus (Fig. 4). No difference in number of survival neurons was observed in dentate gyrus $(F(2,42)=1.520, p=0.230)$. Ceftriaxone treatment after $2 \mathrm{VO}$ showed neuroprotective effects on CA1 and CA3 pyramidal neurons. The numbers of survival neurons in $\mathrm{CA} 1$ and $\mathrm{CA} 3$ were higher in 2VO-CTX group compared with those of $2 \mathrm{VO}-\mathrm{Veh}$ group ( $p=0.020$ and 0.015 , respectively).

\section{Effects of ceftriaxone on hippocampal glutamate transporter-1 (GLT-1) expressions}

There was a trend for the amount of GLT-1 expression to be higher in 2VO-CTX group but this was not statistically significant $(F(2,15)=3.305, p=$ 

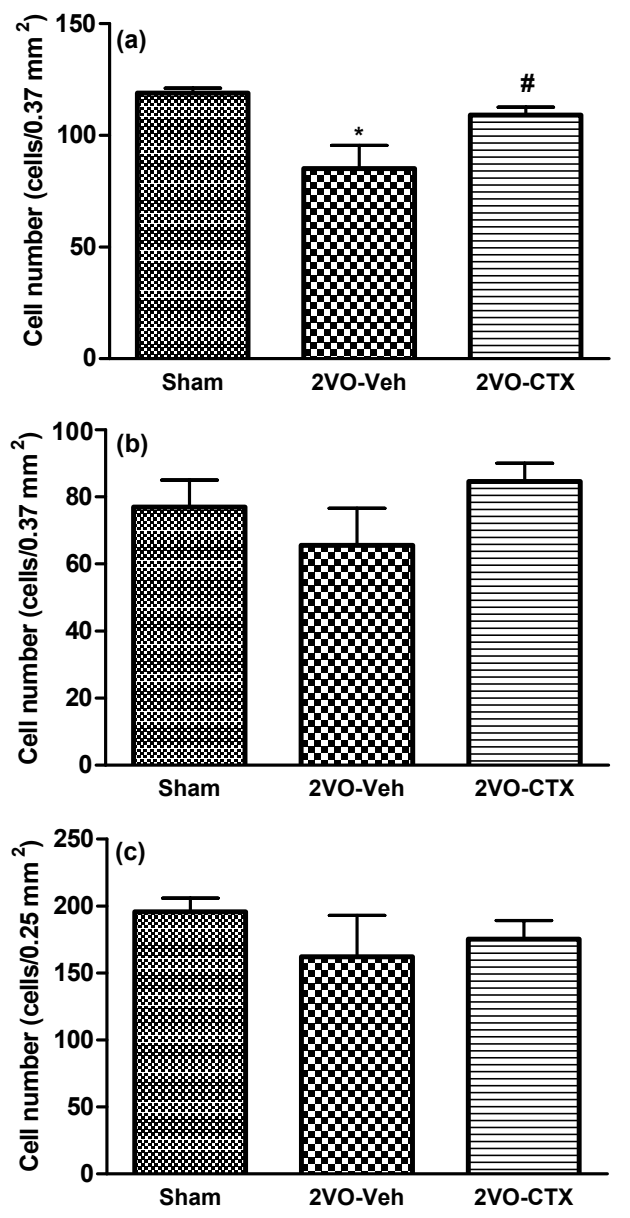

Fig. 3 Effects of ceftriaxone on number of survival hippocampal neuron. Mean hippocampal neuron numbers of (a) CA1 areas, (b) CA3 areas, and (c) dentate gyrus areas, $n=5$. Data present as mean \pm SEM. ${ }^{*} p<0.05$ versus sham-operated group. ${ }^{\#} p<0.05$ versus $2 \mathrm{VO}-$ Veh group.

0.064). The mean amount of GLT-1 was slightly higher in ceftriaxone-treated groups. The percentages of mean hippocampal GLT-1 expression (normalized with mean GLT-1 expression in Sham) were about $186 \%$ and $135 \%$ in $2 \mathrm{VO}-\mathrm{CTX}$ and $2 \mathrm{VO}-\mathrm{Veh}$, respectively (Fig. 5).

\section{DISCUSSION}

Chronic cerebral blood flow reduction has been associated with ageing, mild cognitive impairment and several neurodegenerative diseases including Alzheimer disease and vascular type dementia ${ }^{34-36}$. Bilateral common carotid artery occlusion has been employed in studying the effect of chronic cerebral hypoperfusion because it causes oligaemia, of about $40-65 \%$ of normal CBF. The oligaemic phase can

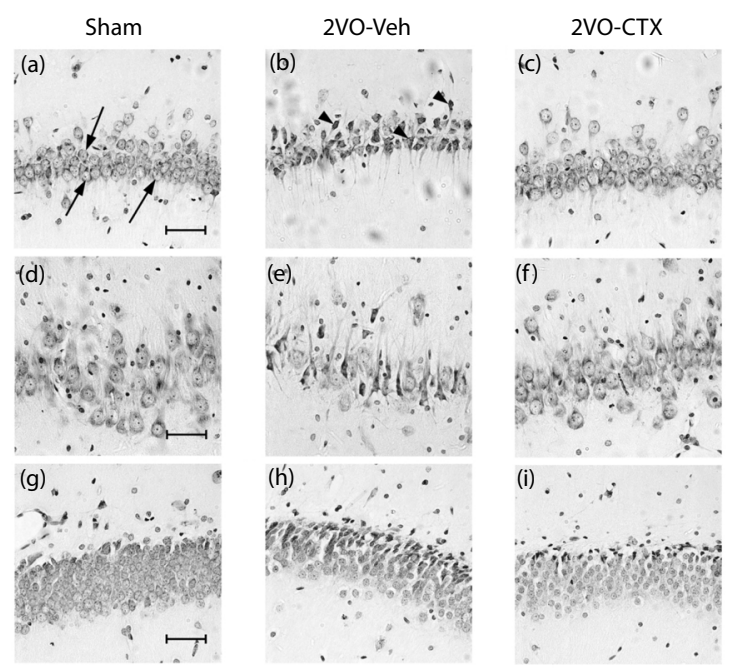

Fig. 4 Effects of ceftriaxone on hippocampal CA1 neuron survival. Photographs show representative cresyl violet staining of (a)-(c) hippocampal CA1 areas, (d)-(f) hippocampal CA3 areas, and (g)-(i) dentate gyrus areas of sham, 2VO-Veh, and 2VO-CTX at $400 \times$ magnification. Live neurons show large nuclei with prominent nucleoli (arrow) while injured or dead neurons are shrinkage with pyknotic appearance (arrow head). Scale bar, $50 \mu \mathrm{m}$.

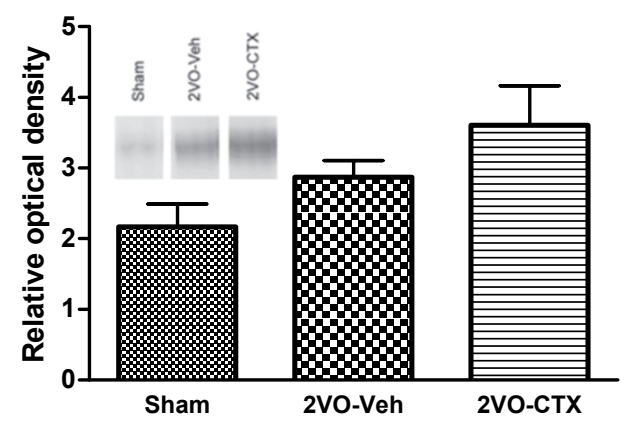

Fig. 5 Effects of ceftriaxone on GLT-1 protein expression in hippocampus. GLT-1 was measured at first week after 2VO induction with $n=6$. Values represent mean \pm SEM.

be maintained long enough to cause neuronal injury and learning/memory deficit ${ }^{37,38}$. Spatial learning and memory impairment without any other sensorimotor deficits have been reported in this model ${ }^{6}$. As shown in our results that bilateral common carotid artery ligation did not affect the performance of the rats in the visible platform session. Besides the visual performance, sensorimotor and motivation are also important for the visible platform session of Morris water maze ${ }^{33}$. The results here also imply 
that both sensorimotor and motivation were also not interrupted.

The mechanism leading to neuronal injury and neurological impairments in chronic cerebral hypoperfusion is still inconclusive. It has been shown in $2 \mathrm{VO}$ model that several cellular functions are altered after cerebral hypoperfusion. The changes in glucose metabolism were detected. At $5 \mathrm{~min}$ after $2 \mathrm{VO}$ onset, adenosine triphosphate (ATP) and phosphocreatine were depleted and sustained at low levels for 2 weeks. Approximately 5 weeks after the onset, ATPase activity was reduced with the increase of lactate concentration in the hippocampus and cerebral cortex. In the end at 8 weeks, ATP level was returned to the normal level. Nevertheless, the level of phosphocreatine remains decrease ${ }^{6,39}$. In transient and focal ischaemic models, massive release of glutamate can induce neuronal injury through various mechanisms including increase intracellular $\mathrm{Ca}^{2+}$, oxidative stress, and inflammation ${ }^{22,40,41}$. The involvement of such glutamate excitotoxicity in chronic cerebral hypoperfusion has been scarcely reported. Therefore, we designed the experiments to evaluate the involvement of glutamate excitotoxicity in chronic cerebral hypoperfusion and to examine whether the rescue of glutamate transporter, GLT-1, would prevent long term effects of cerebral blood flow reduction.

Recently, it was found that $\beta$-lactam antibiotics upregulate glutamate transporters. Among all $\beta$-lactam antibiotics studies, ceftriaxone (CTX) shows the highest potency in the upregulation of glutamate transporter ${ }^{25}$. The upregulation of glutamate transporter by CTX treatment was associated with the reduction of neuronal injury and neurological deficit $^{28,42,43}$. Therefore, we applied CTX to the animals from day 1-5 after vessel ligation. The results showed that CTX treatment improved learning and memory performance measured at 8 weeks after arterial ligation. The mean escape latencies were significantly shorter and the times spent in target quadrant were significantly longer in the CTX-treated rats. We further studied the brain histology after the behavioural assessment. Hippocampus was investigated because of the significant role of this brain area in spatial learning and memory ${ }^{44-46}$. The results showed that CTX treatment attenuated the neuronal injury in hippocampus. The number of survival hippocampal neurons in the CTX-treated rats increased after the arterial ligation especially in CA1 and CA3 areas. Therefore, CTX attenuated hippocampal injury and spatial learning/memory impairment after 2VO.

We hypothesized that CTX may exert neuroprotection by attenuating glutamate excitotoxicity since it has been demonstrated it induces upregulation of glutamate transporters both in vitro and in vivo experiments ${ }^{25,29}$. Glutamate transporters are critical for glutamate removal from the extracellular space ${ }^{47}$. GLT-1 which is specifically expressed in astrocyte is responsible for up to $90 \%$ of glutamate uptake in the brain $^{48}$. GLT-1 protein was measured one week after $2 \mathrm{VO}$ induction, during which time the peak of CTXupregulation of GLT-1 with effective dose of CTX occurs $^{27,43}$. Our results showed that the percentages of mean hippocampal GLT-1 expression were about $186 \%$ for 2 VO-CTX and $135 \%$ for 2 VO-Veh compared with that of the sham. However, the difference among groups was not statistically significant. This could be because of the dynamic characteristic of the $\mathrm{CBF}$ and the pathophysiological processes occur after arterial ligation. Our results agrees with other studies in focal ischaemic and hypoxic-ischaemic model that showed no alteration in glutamate transporter protein level ${ }^{28,43,49}$. Some studies showed increased uptake activity without transporter protein level changes ${ }^{28}$. CBF gradually increases after initial drop immediately after arterial ligation to reach approximately $70 \%$ of control rats within 8 weeks to 3 months ${ }^{50,51}$. Therefore, the GLT-1 expression might also depend on the duration after cerebral hypoperfusion. Interestingly, at 10 weeks of $2 \mathrm{VO}$ that glutamate uptake decreases ${ }^{31}$. Therefore glutamate transporter expression and glutamate uptake activity should be further evaluated to directly answer the involvement of glutamate transporter and glutamate excitotoxicity in chronic cerebral hypoperfusion. Besides, other mechanisms including oxidative stress and inflammatory responses may also contribute to the pathophysiology of chronic cerebral hypoperfusion. Antioxidants, such as green tea polyphenols, and immunosuppressive drugs, such as cyclosporin A and FK-506, have protective effects and attenuated cognitive impairment in $2 \mathrm{VO}$ rats, suggesting the role of oxidative stress and inflammation in chronic cerebral hypoperfusion ${ }^{52-54}$.

In conclusion, the ceftriaxone treatment improved spatial learning and memory performance in the Morris water maze, and increased hippocampal CA1 and CA3 neuronal number in the hippocampus. The results suggest that treatments targeting glutamate excitotoxicity mechanisms may be beneficial during the onset of chronic cerebral hypoperfusion in rats.

Acknowledgements: This study was supported by a Siriraj graduate thesis scholarship, Mahidol University and the strategic scholarships for frontier research network for the Ph.D. programme Thai doctoral degree from office of the higher education commission, Thailand. 


\section{REFERENCES}

1. Kaplan B, Brint S, Tanabe J, Jacewicz M, Wang XJ, Pulsinelli W (1991) Temporal thresholds for neocortical infarction in rats subjected to reversible focal cerebral ischemia. Stroke 22, 1032-9.

2. Lipton P (1999) Ischemic cell death in brain neurons. Physiol Rev 79, 1431-568.

3. Tanaka K, Wada-Tanaka N, Miyazaki I, Nomura M, Ogawa N (2002) Chronic cerebral hypoperfusion induces striatal alterations due to the transient increase of $\mathrm{NO}$ production and the depression of glutathione content. Neurochem Res 27, 331-6.

4. Bennett SAL, Tenniswood M, Chen JH, Davidson CM, Keyes MT, Fortin T, Pappas BA (1998) Chronic cerebral hypoperfusion elicits neuronal apoptosis and behavioral impairment. NeuroReport 9, 161-6.

5. Kim SK, Cho KO, Kim SY (2008) White matter damage and hippocampal neurodegeneration induced by permanent bilateral occlusion of common carotid artery in the rat: Comparison between Wistar and SpragueDawley strain. Kor J Physiol Pharmacol 12, 89-94.

6. Farkas E, Luiten PG, Bari F (2007) Permanent, bilateral common carotid artery occlusion in the rat: a model for chronic cerebral hypoperfusion-related neurodegenerative diseases. Brain Res Rev 54, 162-80.

7. Sopala M, Danysz W (2001) Chronic cerebral hypoperfusion in the rat enhances age-related deficits in spatial memory. J Neural Transm 108, 1445-56.

8. Liu HX, Zhang JJ, Zheng P, Zhang Y (2005) Altered expression of MAP-2, GAP-43, and synaptophysin in the hippocampus of rats with chronic cerebral hypoperfusion correlates with cognitive impairment. Mol Brain Res 139, 169-77.

9. Shibata M, Yamasaki N, Miyakawa T, Kalaria RN, Fujita Y, Ohtani R, Ihara M, Takahashi R, Tomimoto $H$ (2007) Selective impairment of working memory in a mouse model of chronic cerebral hypoperfusion. Stroke 38, 2826-32.

10. Ueda M, Muramatsu H, Kamiya T, Muramatsu A, Mori T, Terashi A, Katayama Y (2000) Pyruvate dehydrogenase activity and energy metabolite levels following bilateral common carotid artery occlusion in rat brain. Life Sci 67, 821-6.

11. He XL, Wang YH, Gao M, Li XX, Zhang TT, Du GH (2009) Baicalein protects rat brain mitochondria against chronic cerebral hypoperfusion-induced oxidative damage. Brain Res 1249, 212-21.

12. de la Torre JC, Aliev G (2005) Inhibition of vascular nitric oxide after rat chronic brain hypoperfusion: spatial memory and immunocytochemical changes. J Cerebr Blood Flow Metabol 25, 663-72.

13. Doyle KP, Simon RP, Stenzel-Poore MP (2008) Mechanisms of ischemic brain damage. Neuropharmacology 55, 310-8.

14. Smith WS (2004) Pathophysiology of focal cerebral ischemia: a therapeutic perspective. J Vasc Intervent
Radiol 15, S3-12.

15. Anitha M, Nandhu MS, Anju TR, Jes P, Paulose CS (2011) Targeting glutamate mediated excitotoxicity in Huntington's disease: neural progenitors and partial glutamate antagonist-memantine. Med Hypotheses 76, 138-40.

16. Estrada Sánchez AM, Mejía-Toiber J, Massieu L (2008) Excitotoxic neuronal death and the pathogenesis of Huntington's disease. Arch Med Res 39, 265-76.

17. Shaw PJ, Ince PG (1997) Glutamate, excitotoxicity and amyotrophic lateral sclerosis. J Neurol 244 Suppl 2, S3-14.

18. Riedel G, Platt B, Micheau J (2003) Glutamate receptor function in learning and memory. Behav Brain Res 140, $1-47$.

19. De Camilli P, Jahn R (1990) Pathways to regulated exocytosis in neurons. Annu Rev Physiol 52, 625-45.

20. Danbolt NC (2001) Glutamate uptake. Progr Neurobiol 65, 1-105.

21. Nie H, Weng HR (2010) Impaired glial glutamate uptake induces extrasynaptic glutamate spillover in the spinal sensory synapses of neuropathic rats. J Neurophysiol 103, 2570-80.

22. Bano D, Nicotera $\mathrm{P}$ (2007) $\mathrm{Ca}^{2+}$ signals and neuronal death in brain ischemia. Stroke 38, 674-6.

23. Kristian T, Siesjo BK (1998) Calcium in ischemic cell death. Stroke 29, 705-18.

24. Simon RP, Griffiths T, Evans MC, Swan JH, Meldrum BS (1984) Calcium overload in selectively vulnerable neurons of the hippocampus during and after ischemia: an electron microscopy study in the rat. J Cerebr Blood Flow Metabol 4, 350-61.

25. Rothstein JD, Patel S, Regan MR, Haenggeli C, Huang YH, Bergles DE, Jin L, Dykes Hoberg M, et al (2005) $\beta$-Lactam antibiotics offer neuroprotection by increasing glutamate transporter expression. Nature 433, 73-7.

26. Bennett PN, Brown JM (2008) Clinical Pharmacology, 10th edn, Churchill Livingstone Cambridge.

27. Chu K, Lee ST, Sinn DI, Ko SY, Kim EH, Kim JM, Kim SJ, Park DK, et al (2007) Pharmacological induction of ischemic tolerance by glutamate transporter-1 (EAAT2) upregulation. Stroke 38, 177-82.

28. Thöne-Reineke C, Neumann C, Namsolleck P, Schmerbach K, Krikov M, Schefe JH, Lucht K, Hörtnagl $\mathrm{H}$, et al (2008) The $\beta$-lactam antibiotic, ceftriaxone, dramatically improves survival, increases glutamate uptake and induces neurotrophins in stroke. $J$ Hypertens 26, 2426-35.

29. Lee SG, Su ZZ, Emdad L, Gupta P, Sarkar D, Borjabad A, Volsky DJ, Fisher PB (2008) Mechanism of ceftriaxone induction of excitatory amino acid transporter2 expression and glutamate uptake in primary human astrocytes. J Biol Chem 283, 13116-23.

30. Uehara H, Yoshioka H, Kawase S, Nagai H, Ohmae T, Hasegawa K, Sawada T (1999) A new model of white matter injury in neonatal rats with bilateral carotid artery occlusion. Brain Res 837, 213-20. 
31. Vicente E, Degerone D, Bohn L, Scornavaca F, Pimentel A, Leite MC, Swarowsky A, Rodrigues L, et al (2009) Astroglial and cognitive effects of chronic cerebral hypoperfusion in the rat. Brain Res 1251, 204-12.

32. He Z, Huang L, Wu Y, Wang J, Wang H, Guo L (2008) DDPH: Improving cognitive deficits beyond its $\alpha_{1}$ adrenoceptor antagonism in chronic cerebral hypoperfused rats. Eur J Pharmacol 588, 178-88.

33. Morris R (1984) Developments of a water-maze procedure for studying spatial learning in the rat. $J$ Neurosci Meth 11, 47-60.

34. Chen JJ, Rosas HD, Salat DH (2011) Age-associated reductions in cerebral blood flow are independent from regional atrophy. NeuroImage 55, 468-78.

35. Schuff N, Matsumoto S, Kmiecik J, Studholme C, Du A, Ezekiel F, Miller BL, Kramer JH, et al (2009) Cerebral blood flow in ischemic vascular dementia and Alzheimer's disease, measured by arterial spin-labeling magnetic resonance imaging. Alzheimers Dement $\mathbf{5}$, 454-62.

36. Johannsen P, Jakobsen J, Gjedde A (2000) Statistical maps of cerebral blood flow deficits in Alzheimer's disease. Eur J Neurol 7, 385-92.

37. Ni J, Ohta H, Matsumoto K, Watanabe H (1994) Progressive cognitive impairment following chronic cerebral hypoperfusion induced by permanent occlusion of bilateral carotid arteries in rats. Brain Res 653, 231-6.

38. Schmidt-Kastner R, Aguirre-Chen C, Saul I, Yick L, Hamasaki D, Busto R, Ginsberg MD (2005) Astrocytes react to oligemia in the forebrain induced by chronic bilateral common carotid artery occlusion in rats. Brain Res 1052, 28-39.

39. Plaschke K (2005) Aspects of ageing in chronic cerebral oligaemia. Mechanisms of degeneration and compensation in rat models. J Neural Transm 112, 393-413.

40. Sugawara T, Fujimura M, Noshita N, Kim GW, Saito A, Hayashi T, Narasimhan P, Maier CM, Chan PH (2004) Neuronal death/survival signaling pathways in cerebral ischemia. NeuroRX 1, 17-25.

41. Giffard RG, Han RQ, Emery JF, Duan M, Pittet JF (2008) Regulation of apoptotic and inflammatory cell signaling in cerebral ischemia: the complex roles of heat shock protein 70. Anesthesiology 109, 339-48.

42. Hota SK, Barhwal K, Ray K, Singh SB, Ilavazhagan G (2008) Ceftriaxone rescues hippocampal neurons from excitotoxicity and enhances memory retrieval in chronic hypobaric hypoxia. Neurobiol Learn Mem 89 , 522-32.

43. Lai PC, Huang YT, Wu CC, Lai CJ, Wang PJ, Chiu TH (2011) Ceftriaxone attenuates hypoxic-ischemic brain injury in neonatal rats. J Biomed Sci 18, 69.

44. Broadbent NJ, Squire LR, Clark RE (2004) Spatial memory, recognition memory, and the hippocampus. Proc Natl Acad Sci USA 101, 14515-20.

45. Burgess N, Maguire EA, O'Keefe J (2002) The human hippocampus and spatial and episodic memory. Neuron
$35,625-41$.

46. Jarrard LE (1993) On the role of the hippocampus in learning and memory in the rat. Behav Neural Biol 60, 9-26.

47. Camacho A, Massieu L (2006) Role of glutamate transporters in the clearance and release of glutamate during ischemia and its relation to neuronal death. Arch Med Res 37, 11-8.

48. Maragakis NJ, Rothstein JD (2004) Glutamate transporters: animal models to neurologic disease. Neurobiol Dis 15, 461-73.

49. Lipski J, Wan CK, Bai JZ, Pi R, Li D, Donnelly D (2007) Neuroprotective potential of ceftriaxone in in vitro models of stroke. Neuroscience 146, 617-29.

50. Otori T, Katsumata T, Muramatsu H, Kashiwagi F, Katayama Y, Terashi A (2003) Long-term measurement of cerebral blood flow and metabolism in a rat chronic hypoperfusion model. Clin Exp Pharmacol Physiol 30, 266-72.

51. Tomimoto H, Ihara M, Wakita H, Ohtani R, Lin J-X, Akiguchi I, Kinoshita M, Shibasaki H (2003) Chronic cerebral hypoperfusion induces white matter lesions and loss of oligodendroglia with DNA fragmentation in the rat. Acta Neuropathol 106, 527-34.

52. Xu Y, Zhang JJ, Xiong L, Zhang L, Sun D, Liu H (2010) Green tea polyphenols inhibit cognitive impairment induced by chronic cerebral hypoperfusion via modulating oxidative stress. J Nutr Biochem 21, 741-8.

53. Wakita H, Tomimoto H, Akiguchi I, Kimura J (1995) Protective effect of cyclosporin A on white matter changes in the rat brain after chronic cerebral hypoperfusion. Stroke 26, 1415-22.

54. Ji HJ, Hu JF, Wang YH, Chen XY, Zhou R, Chen NH (2010) Osthole improves chronic cerebral hypoperfusion induced cognitive deficits and neuronal damage in hippocampus. Eur J Pharmacol 636, 96-101. 